\title{
Defect-induced bandgap narrowing in low-k dielectrics
}

\author{
X. Guo ${ }^{1}$, S. W. King ${ }^{2}$, M. R. Baklanov ${ }^{3}$, J. de Marneffe ${ }^{3}$, V. Afanas' $\mathrm{ev}^{4}$, Y. Nishi ${ }^{5}$ and \\ J. L. Shohet ${ }^{1}$ \\ ${ }^{1}$ Plasma Processing \& Technology Laboratory and Department of Electrical and \\ Computer Engineering, University of Wisconsin-Madison, Madison, WI 53706 \\ ${ }^{2}$ Logic Technology Development, Intel Corporation, Hillsboro, Oregon 97124, USA \\ ${ }^{3}$ IMEC, Leuven, Belgium \\ ${ }^{4}$ Catholic University of Leuven, Leuven, Belgium \\ ${ }^{5}$ Stanford University, Stanford, CA 94305
}

\begin{abstract}
:
In this work, core-level X-ray photoelectron spectroscopy was utilized to determine the surface bandgap for various porous and non-porous low-k a-SiCOH dielectrics before and after ion sputtering by examining the onset of inelastic energy loss in core-level atomic spectra. Bandgap narrowing was found in $\mathrm{Ar}^{+}$ion sputtered low-k dielectrics. The reduction of the bandgap ranges from 1.3 to $2.2 \mathrm{eV}$ depending on the film composition. We show that the bandgap narrowing in these low-k dielectrics is produced by the uplifting of the valence-band tail as evidenced by the presence of additional electronic states above the valence-band maximum. Electron-spin-resonance measurements were made on a-SiCOH films to examine the defects in the materials. The localization of each type of defect within the dielectric gap is analyzed and compared. A combination of these measurements with those for the narrowed band gap suggests that the additional electron states generating the narrowed bandgap originate from carbon-related defects in the
\end{abstract}


material. 
Electrical reliability in $\mathrm{Cu}$ interconnect structures, such as stress-induced leakage currents, dielectric-breakdown voltages, and time-dependent dielectric breakdown (TDDB) failures, has become of vital concern as the nano-electronics industry moves to sub-16 $\mathrm{nm}$ and beyond technology nodes and strives to implement interlayer dielectric (ILD) materials with increasingly lower dielectric constants (i.e., low-k). ${ }^{1,2,3}$ These reliability issues are typically attributed to the presence and creation of electrical "traps" or "defects" from downstream porogen removal and plasma etching processes that expose the low-k material to intense ultraviolet (UV) and vacuum ultraviolet (VUV) photons, energetic ions, and chemically active radicals. ${ }^{4,5,6,7}$ The arising bulk inter-gap traps or defect states can affect electron transport across the low-k dielectric thin film significantly. Numerous electrically based measurements have shown a direct correlation between trap/defect states, leakage current, breakdown voltages, and TDDB failures of low-k materials. ${ }^{3,8,9}$ In most cases, the conduction mechanisms in dielectric materials are fundamentally dependent on accurate knowledge of the depths and locations of trap states within the band gap. While due to the loss of translational symmetry of electronic states, there is no "true" bandgap for amorphous materials. The energy levels/positions of the defects within the amorphous low-k dielectrics and their correction to electrical reliability are not clear. To address this issue, a number of empirical measurements have recently been devoted to determine the effective bandgap energy and to delineate the location of extended gap/defect states of the low-k dielectrics. ${ }^{10,11,12}$ Grill et al. had reported bandgap energies of low-k amorphous organosilicate dielectric (a-SiCOH) thin films between 4.74 and $5.92 \mathrm{eV}$, depending on the dielectric constant. ${ }^{10}$ By examining the onset of inelastic energy loss in core-level atomic spectra using X-ray photoelectron spectroscopy (XPS), 
Nichols et al. had measured bandgap of $7.2 \mathrm{eV}$ and $7.7 \mathrm{eV}$ for as-deposited and UV-cured a-SiCOH thin films, respectively. ${ }^{11}$ Using reflection electron energy loss spectroscopy (REELS), King et al. reported the surface band gap to be $8.2 \mathrm{eV}$ for non-porous a-SiCOH $(\mathrm{k}=2.8-3.3)$ and $7.8 \mathrm{eV}$ for porous a-SiCOH $(\mathrm{k}=2.3) .{ }^{12}$

The bandgap energy provides a necessary reference for understanding the depths of trap/defect states within the band gap as well as interfacial energy-barrier heights. Conversely, the creation of defects and vacancies within the band gap can cause a reduction of the band gap by formation of additional electronic states either above the valence band or below the conduction band that overlap with one of these bands. For example, a conduction-band tail state may arise and extend below the conduction-band minimum (CBM) with the presence of vacancies and defect centers near the conduction band edge, or the valence-band maximum (VBM) may uplift due to an excess of electronic states originating nearby from disorders and imperfections of the materials. Both of these can lead a decrease in the band gap. A number of electron-spin resonance (ESR) measurements of porous/non-porous low-k dielectrics have shown that defects within these materials are silicon dangling bonds, neutral or positively charged oxygen vacancies and carbon residues, ${ }^{13,14,15}$ all of which give deep states near the mid band gap. ${ }^{16}$ In addition, the Si-Si dangling bond can generate a bonding state in the lower gap and an antibonding state near the CBM. Oxygen vacancies possess a "shallow" bound state 1-2 eV below the CBM, while non-bridging oxygen can introduce states just above the VBM. ${ }^{16}$ The effects of these additional electronic states on the experimental bandgap of low-k dielectrics are largely unknown and thus a more fundamental knowledge of the bandgap energy of the low-k dielectrics with intrinsic and/or post processing induced 
defect centers is therefore needed. Considering this, an examination and comparison of the surface bandgap were conducted on various porous and non-porous a-SiCOH thin films before and after in-situ Ar+ sputtering in this work, to investigate the band-gap narrowing phenomenon in low-k dielectrics.

Table I provides a summary of the low-k dielectrics under study, which are all aSiCOH thin films deposited with plasma-enhanced chemical-vapor deposition (PECVD) on $300 \mathrm{~mm}$ diameter (100) Si wafers. Neither e-beam nor ultraviolet thermal-processing was utilized after deposition. As can be seen, the a-SiCOH materials examined involve various compositions and dielectric constants that are representative of ILDs currently utilized or being considered for future applications. After the films were produced, XPS measurements were made on the surface. These measurements were used to obtain the bandgap energy by extrapolating a linear fit to the leading edge of the electron energy loss spectra and by locating the onset of inelastic energy loss relative to the core-level peak. ${ }^{11}$ The accuracy of this method can be usually $0.5 \mathrm{eV}$ or less if the electron density in the valence band near surface is laterally homogeneous and the largest source of error is due to uncertainty in the process of linear extrapolation. ${ }^{11}$ During the measurement, the pass energy of the energy analyzer was set to $21 \mathrm{eV}$ with a corresponding absolute resolution of $0.5 \mathrm{eV}$. Each sample was measured 10 times and for each measurement the core-level XPS spectroscopy was scanned 50 times. After that, a $4 \mathrm{keV} \mathrm{Ar}^{+}$ion sputtering beam, from the monatomic argon ion sources equipped in the same analyzing chamber, was utilized in-situ to induce surface defects, because ion sputtering has been shown to be a powerful damage source for a-SiCOH and can create various defects with in the dielectrics effecively. ${ }^{12}$ The ion beam current was $15 \mu \mathrm{A} / \mathrm{cm}^{2}$ and the sputtering time was 
30 seconds. XPS measurements with the same procedure were then performed on the ionsputtered spot of the sample to get a comparison to the bandgap energy before and after ion sputtering.

Figure 1 shows the inelastic-loss spectra near the $\mathrm{O}$ 1s core-level peak collected from a $100 \mathrm{~nm}$ a-SiCOH film $(\mathrm{k}=3.3)$ both before and after in-situ $\mathrm{Ar}^{+}$ion sputtering, which had been calibrated with reference to the $\mathrm{C} 1 \mathrm{~s}$ peak at $284.8 \mathrm{eV}$ to eliminate surfacecharge effects and the location of the $\mathrm{O}$ 1s core-level peak was determined to be 532.6 $\mathrm{eV}$. It should be noted that though any core-level spectra of the material will exhibit the same inelastic losses, the O 1s core-level XPS peak was chosen here because of its higher cross section for absorption of X-ray photons and correspondingly larger photoelectron intensity, so that a sufficient signal-to-noise ratio can be achieved with suitably high resolution. By tracking the intersection of the leading edge (solid straight line) and the background level (dashed horizontal line) of the electron energy inelastic loss spectra, as shown in Figure 1, the onset of inelastic losses of a-SiCOH film $(\mathrm{k}=3.3)$ was found to occur at $540.7 \mathrm{eV}$ before ion sputtering, and $539.4 \mathrm{eV}$ after ion sputtering. To quantify the uncertainties of the linear extrapolation, least-square-fitting regression lines with $95 \%$ confidence were constructed (dotted straight lines) to the inelastic loss peak leading edge. By taking the upper and lower bounds of the confidence intervals and finding their intersection with the background level, the errors on linear extrapolations were estimated to be $\pm 0.3 \mathrm{eV}$ with $95 \%$ confidence. According to these analyses, the bandgap of pristine a-SiCOH film $(\mathrm{k}=3.3)$ was calculated to be $8.1 \pm 0.3 \mathrm{eV}$ by subtracting the binding energy of $\mathrm{O} 1 \mathrm{~s}$ peak at $532.6 \mathrm{eV}$ from that of onset of inelastic losses. This value agrees with the reported band gap energy of $8.2 \mathrm{eV}$ for a-SiCOH film $(\mathrm{k}=3.3)$ measured by 
REELS. ${ }^{12}$ Similarly, the bandgap of the a-SiCOH film $(k=3.3)$ after ion sputtering was calculated to be $6.8 \pm 0.3 \mathrm{eV}$, implying that a bandgap reduction of $1.3 \mathrm{eV}$ occurred to the film after ion sputtering. To further confirm this phenomenon, bandgap measurements using the same procedure were conducted on all of the samples under study and the results are summarized in Table II. As can be seen from Table II, after $4 \mathrm{keV}$ ion-beam sputtering, the bandgap energy of the a-SiCOH films were all reduced and the reduction ranges from $1.3 \mathrm{eV}$ to $2.2 \mathrm{eV}$ depending on the film composition/porosity. The largest reduction of $2.2 \mathrm{eV}$ for the bandgap energy was observed in the a-SiCOH $(\mathrm{k}=2.2)$ film with a higher carbon atomic concentration than others.

According to the uncertainty interval obtained above, the measured narrowing of the bandgap cannot be ascribed to measurement errors alone and suggests that additional states must have been created within the band gap of the ion sputtered a-SiCOH film, as discussed previously. Thus, valence-band XPS were made on the low-k a-SiCOH films ( $k=3.3)$ before and after ion sputtering to examine the density of states of $/$ near the valence band of the a-SiCOH film, as shown in Figure 2a. By linearly fitting to the rising edge of the valence-band spectra, the valence-band edge of the pristine a-SiCOH film was determined to be approximately $4.1 \mathrm{eV}$, which is reasonable for undoped intrinsic a$\mathrm{SiCOH}$ with a measured bandgap of $8.1 \mathrm{eV}$. For the ion sputtered sample, however, additional electronic states were detected above the valence band maximum, resulting in a shift of the valence band edge (VBE) towards to $2.8 \mathrm{eV}$, as illustrated by the arrow in Figure 2a. A schematic representation of the density of states is depicted in Figure 2b on the basis of these results, showing the changes of band structures after ion sputtering. One can see that in the ion-sputtered sample, a valence band tail appears and extends above 
the valence band maximum, thus up-shifting the valance-band edge. By subtracting the measured VBE value of $2.8 \mathrm{eV}$ in the ion-sputtered sample from the $4.1 \mathrm{eV}$ in a pristine sample, an uplift of the VBE was calculated to be $1.3 \mathrm{eV}$ for ion-sputtered a-SiCOH, which is in excellent agreement with the value of bandgap reduction, suggesting that the decrease in the bandgap energy after ion sputtering would originate in the additional electronic states near the valence band, rather than the conduction band.

To obtain more insight on the intrinsic defects generated during ion sputtering, electron spin resonance (ESR) measurements were carried out on a-SiCOH thin films deposited on high-resistivity silicon wafers. Figure 3 shows the conventional first derivative absorption spectra of the ESR signal from the low-k a-SiCOH film $(k=3.3)$ with the applied magnetic field $\mathbf{B}$ parallel with the sample surface. Values of $g$-factor and linewidth of the defect center were determined by fitting the ESR spectra into the Lorentzian derivatives line shape using least-squares. Control spectra were measured on the pristine samples and were observed to exhibit only signals originating from silicon dangling bonds, as illustrated in Figure 3. These silicon dangling bonds, generally known as $\mathrm{P}_{\mathrm{b} 0}$ and/or $\mathrm{P}_{\mathrm{b} 1}$ centers, are usually from the underlying low-k a-SiCOH/Si interface with no measurable density of paramagnetic defects pertaining to the low-k dielectric layer itself. However, after ion sputtering, the number of silicon dangling bond defects increased in ion sputtered a-SiCOH, implying the creation of surface silicon dangling bond defects similar to the bulk E' centers. ${ }^{17,18}$ In addition, peaks with much higher intensity emerged in the ESR spectra, indicating generation of new defects in the a$\mathrm{SiCOH}$ film. The predominant peak is characteristic with a $g$-factor of 2.0006 and a linewidth of $2 \mathrm{GHz}$, which has been attributed to surface oxygen vacancies (SOV) via 
depleting a-SiCOH surface of oxygen during $\mathrm{Ar}^{+}$sputtering. The other signal at $\mathrm{g}=$ 2.0026 with an approximate linewidth of $7 \mathrm{GHz}$ is identified as due to carbon dangling bonds back-bonded to $\mathrm{C}$ or $\mathrm{Si}$ atoms. ${ }^{14}$

These observations are consistent with the ESR results reported by previous authors that ion sputtering-induced defects in low-k dielectrics are oxygen/silicon vacancies analogous to the oxygen-deficient centers/silicon dangling bond centers in bulk $\mathrm{SiO}_{2}$ and carbon dangling bonds from organic residues in the material due to inefficient extraction of the second phase organic pore building material. ${ }^{14,15}$ The surface oxygen vacancy (SOV) has been previously identified as a defect that creates sub-gap surface defects at $\approx$ 5.0 and $7.2 \mathrm{eV},{ }^{12}$ which are in the upper half of the band gap and close to the conduction band minimum. While the silicon dangling bounds possess a highly localized state near the mid-band gap, lying near the isolated $s p^{3}$ or $p$ hybrid energies, which will not contribute to the states below the Fermi level.${ }^{16}$ As a result, additional electron states near the valence-band maximum must be attributed to the carbon-related defects created during ion sputtering, which can give rise to "deep" energy levels near the valence band in the bandgap of the $\mathrm{SiO}_{2}$-like skeleton of a low-k dielectric material. For example, the excessive carbon-dangling bonds at the interfaces results in electron states distributed in the lower half of the oxide bandgap; also electron states associated with a-C:H layers can be found in the same energy range. ${ }^{19,20}$

On the other hand, the bandgap narrowing actually is related to the amount of carbon in the films. The largest bandgap reduction of $2.2 \mathrm{eV}$ occurred in those a-SiCOH films $(\mathrm{k}=2.2)$ that exhibit the highest concentration of carbon dangling bonds, as shown by the highest intensity ESR signal ( $\mathrm{g}=2.0026$ ) after ion sputtering. Hence, it seems logical to 
conclude that the carbon-related defects are responsible for the bandgap narrowing in a$\mathrm{SiCOH}$ films. However, it is still important to note that for $\mathrm{Ar}^{+}$sputtered samples, the detected carbon-related ESR signal exhibits a linewidth of approximate $7 \mathrm{GHz}$, pointing to a more complex structure of this defect. Due to the inability to detect isotopic hyperfine interactions in the ESR spectra, more experimental and theoretical work will be needed to fully determine the impact of the carbon-related defects on the electronic characteristics of low-k materials.

To conclude, core-level XPS measurements have been utilized to measure the band gap energy of low-k a-SiCOH films before and after $\mathrm{Ar}^{+}$ion sputtering. The results show that bandgap narrowing occurs in ion-sputtered a-SiCOH films and the reduction of bandgap varies with the film composition. Valence-band XPS measurements show additional electron states are created near the valence band by ion sputtering and the bandgap reduction originates from the tail extended above the valence-band maximum. It is likely that the resulting defects are carbon dangling-bond defects from organic residues in the material. These observations suggest that the defects within the band gap of the low-k dielectric films are largely responsible for the bandgap narrowing and likely limit the dielectric reliability. Though these results are based on ion-sputtered low-k a-SiCOH films, they show that defects within the films can reduce the band gap energy and that carbon-related defects are responsible for this. Compared with plasma processing and/or VUV irradiation, ion sputtering is likely to be a more damaging source to a-SiCOH. However, plasma processing and VUV irradiation can create similar defects within aSiCOH. Thus, these measurements help us understand the electrical degradation mechanisms after processing alone. Valence-band XPS measurements show additional 
electron states are created near the valence band by ion sputtering and the bandgap reduction originates from the tail extended above the valence-band maximum. It is likely that the resulting defects are carbon dangling-bond defects from organic residues in the material,. These observations suggest that the defects within the band gap of the low-k dielectric films are largely responsible for the bandgap narrowing and likely limit the dielectric reliability.

This work was supported by the Semiconductor Research Corporation under Contract 2012-KJ-2359 and by the National Science Foundation under Grant CBET-1066231. 


\section{Table Captions}

Table I. Summary of properties for PECVD low-k a-SiCOH thin films investigated in this study.

Table II. Summary of bandgap energy and valence band position before and after ion sputtering for the $\mathrm{SiCOH}$ films under study. 


\section{Figure Captions}

Figure 1. Bandgap measurements of $\mathrm{SiCOH}(\mathrm{k}=3.3)$ film before and after ion sputtering by linearly fitting to electron energy loss spectra with $95 \%$ confidence interval.

Figure 2. (a) Valence band XPS spectra of $\mathrm{SiCOH}(\mathrm{k}=3.3)$ film before and after ion sputtering, where the " 0 " binding energy corresponds to the energy of the Fermi level. Thin black lines show the linear extrapolation of the curves used for deriving the band edge position; (b) schematic representation of the density of states of $\mathrm{SiCOH}(\mathrm{k}=3.3)$ film before and after ion sputtering.

Figure 3. ESR signals measured on $\mathrm{SiCOH}(\mathrm{k}=3.3)$ film before and after ion sputtering. 


\section{TABLES}

Table I.

TABLES

\begin{tabular}{ccccc}
\hline \hline Film & $\begin{array}{c}\text { k-Value } \\
( \pm 0.1)\end{array}$ & $\begin{array}{c}\text { Refractive Index } \\
( \pm 0.1)\end{array}$ & $\begin{array}{c}\text { Density } \\
\left(\mathrm{g} / \mathrm{cm}^{3}\right)\end{array}$ & $\begin{array}{c}\text { Porosity } \\
(\%)\end{array}$ \\
\hline $\mathrm{a}^{-} \mathrm{SiC}_{0.4} \mathrm{O}_{1.2} \mathrm{H}$ & 3.3 & 1.44 & $1.5 \pm 0.1$ & 0 \\
$\mathrm{a}-\mathrm{SiC}_{1.1} \mathrm{O}_{1.7} \mathrm{H}$ & 2.5 & 1.38 & $1.3 \pm 0.1$ & 25 \\
$\mathrm{a}-\mathrm{SiC}_{1.4} \mathrm{O}_{1.6} \mathrm{H}$ & 2.2 & 1.32 & $1.1 \pm 0.1$ & 35 \\
\hline \hline
\end{tabular}

Table II.

TABLES

\begin{tabular}{ccccc}
\hline \hline \multirow{2}{*}{ Film } & \multicolumn{2}{c}{ Before ion sputtering } & \multicolumn{2}{c}{ After ion sputtering } \\
\cline { 2 - 5 } & $\begin{array}{c}\text { Band gap } \\
( \pm 0.3 \mathrm{ev})\end{array}$ & $\begin{array}{c}\text { Valence band } \\
\text { edge }( \pm 0.1 \mathrm{ev})\end{array}$ & $\begin{array}{c}\text { Band gap } \\
( \pm 0.3 \mathrm{ev})\end{array}$ & $\begin{array}{c}\text { Valence band } \\
\text { edge }( \pm 0.1 \mathrm{ev})\end{array}$ \\
\hline $\mathrm{a}-\mathrm{SiC}_{0.4} \mathrm{O}_{1.2} \mathrm{H}$ & 8.1 & 4.1 & 6.8 & 2.8 \\
$\mathrm{a}-\mathrm{SiC}_{1.1} \mathrm{O}_{1.7} \mathrm{H}$ & 8.0 & 4.0 & 6.2 & 2.2 \\
$\mathrm{a}-\mathrm{SiC}_{1.4} \mathrm{O}_{1.6} \mathrm{H}$ & 8.3 & 4.2 & 6.1 & 2.0 \\
\hline \hline
\end{tabular}




\section{FIGURES}

Figure 1.

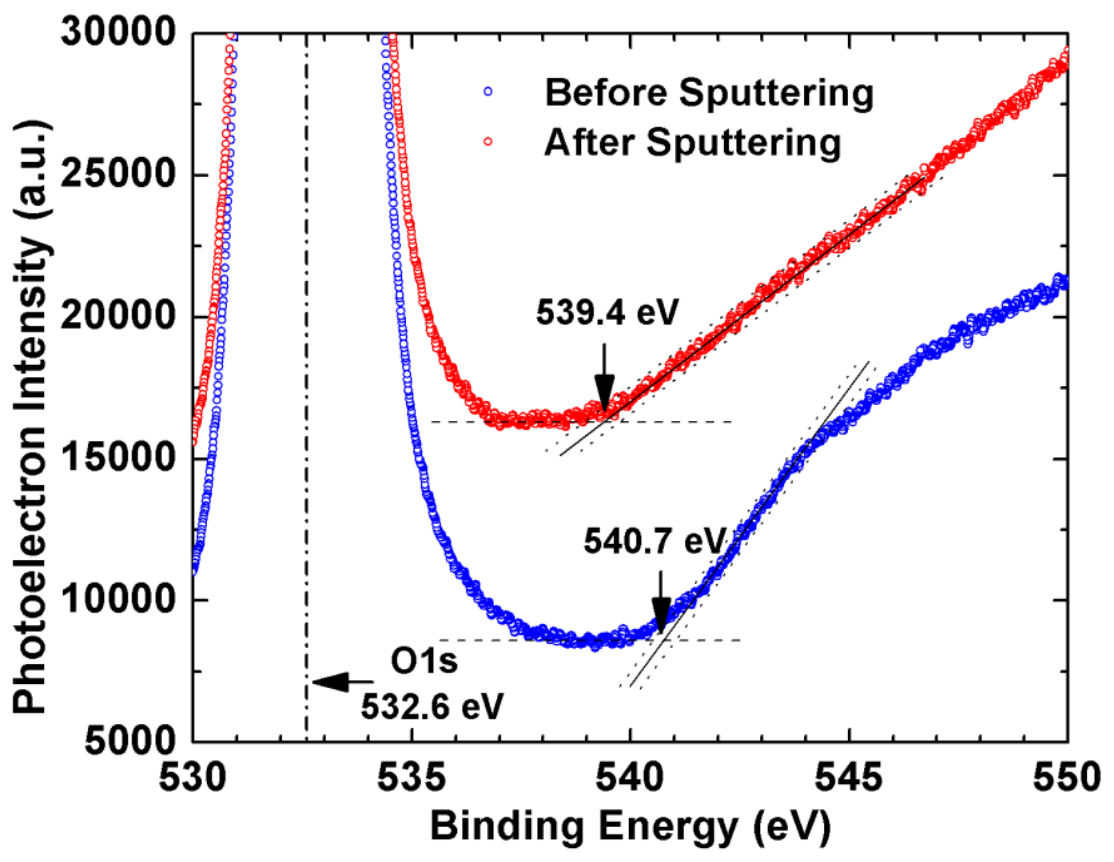


FIGURES

Figure 2.
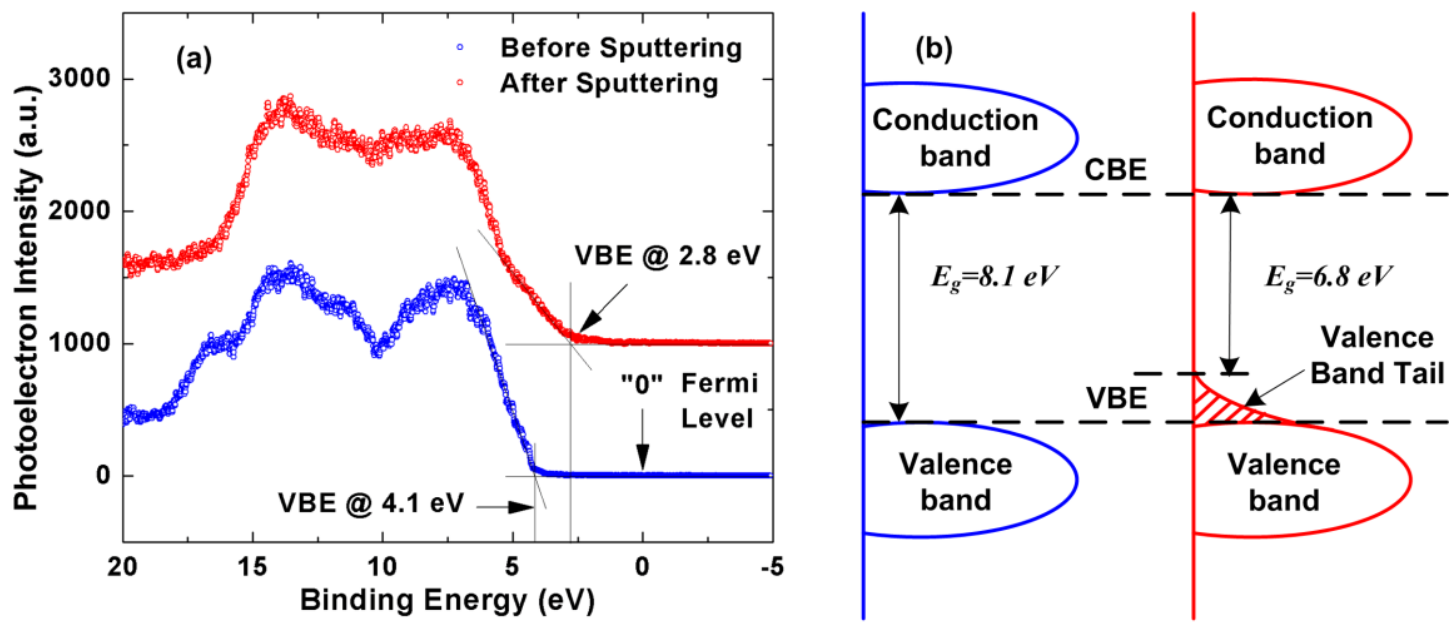

16 


\section{FIGURES}

Figure 3.

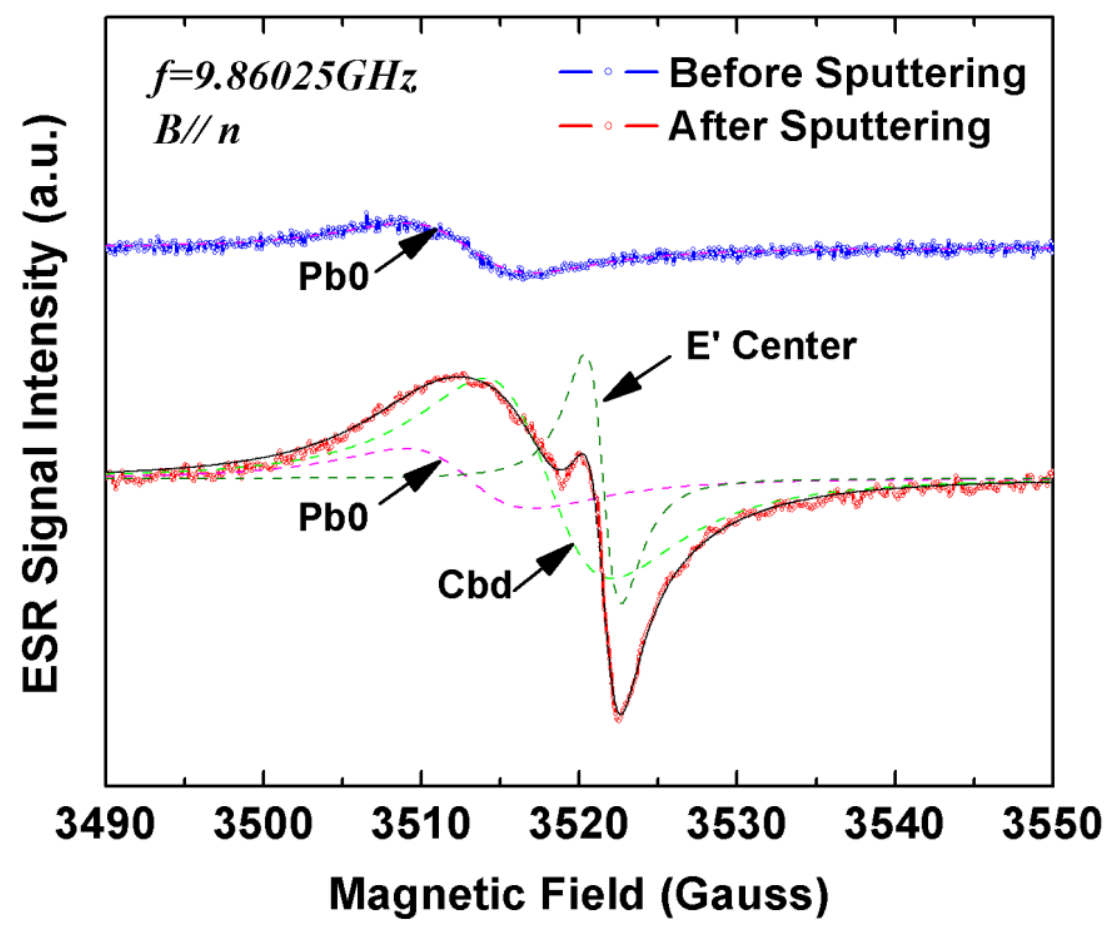




\section{REFERENCES}

${ }_{1}^{1}$ J. Lloyd, E. Liniger, and T. Shaw, J. Appl. Phys. 98, 84109 (2005).

${ }^{2}$ J. Atkin, E. Cartier, T. Shaw, J. Lloyd, R. Laibowitz, and T. Heinz, Microelectron. Eng. 86, 1891 (2009).

${ }^{3}$ M. Nichols, H. Sinha, C. Wiltbank, G. Antonelli, Y. Nishi, and J. Shohet, Appl. Phys. Lett. 100, 112905 (2012).

${ }^{4}$ F. Chen and M. Shinovsky, IEEE Trans. Elect. Dev. 56, 2 (2009).

${ }^{5}$ K. Tanbara, and Y. Kamigaki. J. Electron. Chem. Soc. 157, G95 (2010).

${ }^{6}$ X. Guo, J. E. Jakes, S. Banna, Y. Nishi, and J. L. Shohet, J. Appl. Phys.116, 044103 (2014).

${ }^{7}$ X. Guo, S. W. King, H. Zheng, P. Xue, Y. Nishi, and J. L. Shohet, Appl. Phys. Lett. 106, 012904 (2015).

${ }^{8}$ B. Bittel, P. Lenahan, and S. King, Appl. Phys. Lett. 97, 63506 (2010).

${ }_{9}^{9}$ J. Atkin, D. Song, T. Shaw, E. Cartier, R. Laibowitz, and T. Heinz, J. Appl. Phys. 103, 94104 (2008).

10 A. Grill, J. Appl. Phys. 93, 1785 (2003).

${ }^{11}$ M. T. Nichols, W. Li, D. Pei, G. A. Antonelli, Q. Lin, S. Banna, Y. Nishi, and J. L. Shohet, J. Appl. Phys. 115, 094105 (2014).

${ }^{12}$ S. W. King, B. French, and E. Mays, J. Appl. Phys. 113, 044109 (2013).

${ }^{13}$ H. Ren, M. T. Nichols, G. Jiang, G. A. Antonelli, Y. Nishi, and J. L. Shohet, Appl. Phys. Lett. 98, 102903 (2011).

${ }^{14}$ V. V. Afanas'ev, A. P. D. Nguyen, M. Houssa, A. Stesmans, Zs. Tokei, and M. R. Baklanov, Appl. Phys. Lett. 102, 172908 (2013). 
${ }_{15}$ T. A. Pomorski, B. C. Bittel, P. M. Lenahan, E. Mays, C. Ege, J. Bielefeld, D. Michalak, and S. W. King, J. Appl. Phys. 115, 234508 (2014)

${ }^{16}$ E. P O'Reilly and J. Roberson, Phys. Rev. B 27, 3780 (1983).

${ }_{17}$ J. Thomas and S. Hofmann, J. Vac. Sci. Technol. A 3, 1921 (1985).

${ }^{18}$ E. Paparazzo, J. Phys. D 20, 1091 (1987).

${ }^{19}$ V. V. Afanas'ev, M. Bassler, G. Pensl, M. Schulz, and E. Stein von Kamienski, J. Appl. Phys. 79, 3108 (1996).

${ }^{20}$ V. V. Afanas'ev, A. Stesmans, and M. O. Andersson, Phys. Rev. B 54, 10820 (1996). 